\title{
Factores familiares y sociales de alto riesgo asociados al trabajo infantil en ciudades de la Costa Caribe colombiana*
}

\author{
High risk family and social factors associated with child \\ labour in cities of the colombian caribbean coast
}

Recibido: marzo 8 de 2010 | Revisado: septiembre 11 de 2010 | Aceptado: abril 15 de 2011

\author{
VANESSA ROMERO MENDOZA ** \\ JOSÉ JUAN AMAR AMAR **** \\ JoRge PALACIO** \\ CAmilo Madariaga Orozco* \\ ElOÍSA SIERRA CRISSON ${ }^{* * * * * * *}$ \\ SANDRA MiLENA QUINTERO GONZÁLEZ \\ Universidad del Norte, Barranquilla, Colombia
}

SICI: 1697-9267(201206)11:2<481:FFYSAR>2.0.TX;2-O

Para citar este artículo. Romero, V., Amar, J. J., Palacio, J., Madariaga, C., Sierra, E. \& Quintero, S. M. (2012). Factores familiares y sociales de alto riesgo asociados al trabajo infantil en ciudades de la Costa Caribe colombiana. Universitas Psychologica, 11(2), 481-496.

Resultados preliminares del proyecto "Eliminación del trabajo infantil a través de la educación en Colombia" (2008), desarrollado por PARTNERS of the AMERICAS, CINDE, DevTech y Mercy Corps., con el apoyo del Ministerio de Trabajo de Estados Unidos (DOL-IL16574-07-75K). La Fundación Mamonal (Cartagena) y la Fundación para el Desarrollo del Niño, la Familia y la Comunidad (Santa Marta).

** Docente catedrático, Grupo de Investigación en Desarrollo Humano - GIDHUM. E-mail: vmromero@uninorte.edu.co

**** Ph.D. Decano de la División de Humanidades y Ciencias Sociales.E-mail: jamar@uninorte.edu.co

***** Docente del Departamento de Psicología. E-mail: jpalacio@uninorte.edu.co

******* Coordinador de Proyectos especiales y Docente del Departamento de Psicología. E-mail: cmadaria@uninorte.edu.co. ResearcherID: Madariaga, C. F-4291-2012.

******** E-mail: emsierra@uninorte.edu.co

********* E-mail: smquintero@uninorte.edu.co

\section{RESUMEN}

Se describen los factores familiares y sociales de alto riesgo asociados al trabajo infantil. La muestra, de carácter intencional, estuvo constituida por 835 niños, niñas y adolescentes entre 6 y 17 años $(M=10.6$ años y $D E=2.2$ ) de las ciudades de Barranquilla, Santa Marta y Cartagena, que participaron en el proyecto "Edúcame primero, Colombia" durante 2008. Los resultados presentan datos relevantes sobre el estado sociodemográfico de los niños participantes, sus características familiares y condiciones sociales, que permitirán establecer una línea base actualizada y abrir camino para la construcción de estrategias de intervención efectivas sobre un flagelo que azota a los niños, niñas y jóvenes no solo de Colombia, sino del mundo.

Palabras clave autores

Trabajo infantil, factores de riesgo, familia, niños, Colombia.

Palabras clave descriptores

Región caribe, psicología social, diseño descriptivo, investigación cuantitativa.

\section{A B S T R A C T}

The investigation describes the familiar and social factors of high risk associated with child labour. The sample of intentional character, was made up of 835 children and teenagers between 6 and 17 years (average of 10.6 years and DT $=2.2$ ) of the cities of Barranquilla, Santa Marta, and Cartagena, which participated in the project "Educate First Your Colombia "during the year 2008. The results presented relevant data on the State population partner participating children, familiar characteristics and social conditions, to help establish a baseline updated and pave way for the construction of effective intervention strategies on a scourge that flogs the children and young people not only of Colombia but worldwide.

Key words authors

Child labour, risk factors, family, children, Colombia.

Key words plus

Cariben region, social psychology, descriptive design, quantitative research. 


\section{Introducción}

En Colombia, se han presentado cifras que señalan una reducción significativa del trabajo infantil entre los años 2001 y 2007, indicando que se pasó de 1.409.777 niños, niñas y adolescentes en el mercado laboral en el 2001, hasta llegar a 786.567 en el 2007, con una reducción total de 623.210. Esto tiene una explicación en el crecimiento económico del país durante este periodo, unido al incremento de la cobertura educativa (Departamento Administrativo Nacional de Estadística [DANE], 2008). Los niños que seguían realizando actividades laborales eran aquellos que estaban en la economía informal y las políticas del Gobierno no lograban alcanzar. Estas cifras eran muy optimistas, pero del 2008 hasta el momento, las expectativas de desarrollo del país se han visto disminuidas por una gran variedad de factores, entre los que resaltan las dificultades en las relaciones con los países vecinos y los cambios ambientales que han alterado los procesos de producción habituales. Esta situación está generando mayores niveles de pobreza, que se traduce muy probablemente en un incremento de los niños, niñas y adolescentes (NNA) que ingresan o retornan al mercado laboral. De esta manera, los NNA trabajadores se hacen cada vez más visibles, sobre todo en las ciudades de los países en vías de desarrollo, al igual que los programas dirigidos a prevenir o atacar este flagelo.

La información sobre la efectividad de estos programas y sobre el impacto que tienen en la población infantil es aún muy escasa y dispersa, lo cual se entiende por ser una problemática multideterminada que se incluye en los malos tratos a la infancia (Herce \& Torres, 1996), pero se hace necesario y urgente rescatar información actualizada sobre los factores de riesgo o protectores a nivel familiar o social, que permitan atacar mejor este problema. De allí que en esta investigación se pretenda describir los principales factores familiares y sociales de alto riesgo asociados a NNA que trabajan o que están en alto riesgo de hacerlo en las ciudades de Barranquilla, Santa Marta y Cartagena, que participaron en el proyecto "Edúcame primero, Colombia" durante el año 2008.

\section{El trabajo infantil: definición, contexto e intervención}

El Comité Interinstitucional para la Erradicación del Trabajo Infantil (2003) define la Explotación Laboral Infantil o Trabajo Infantil (TI) como toda actividad de comercialización, producción, transformación, distribución o venta de bienes o servicios, remunerada o no, realizada en forma independiente o al servicio de otra persona natural o jurídica, por personas que no han cumplido los 18 años de edad. Este fenómeno se ha incrementado en el mundo por diversas razones que van desde gobiernos débiles en sus instituciones, hasta desastres naturales y cambios climáticos que desarticulan brutalmente a las familias y a la sociedad. Según la Organización Internacional del Trabajo (OIT, 2008), en el mundo uno de cada siete niños se encontraba realizando algún tipo de trabajo infantil, en particular en África Subsahariana, donde el porcentaje de niños ocupados en actividades económicas es el más alto de todas las regiones del mundo, alcanzando el $26 \%$, lo que representa cerca de 50 millones de niños. En el caso de Latinoamérica, las cifras presentan entre el 2000 y 2004 una disminución del $11 \%$ en el número de niños que trabajan.

En Colombia, la OIT y el Programa Internacional de Erradicación del Trabajo Infantil (IPEC) estiman, en el 2005, que unos 2.318.378 de NNA trabajan como agricultores, albañiles, lecheros, empleados domésticos, voceadores de periódicos, limpiabotas, vendedores o limpiadores en los semáforos, entre otras labores, convirtiéndose en personajes característicos de nuestras ciudades. Su presencia se incrementó por el proceso de industrialización del país en el siglo XX, pero sobre todo por la permisividad, indiferencia y aceptación de la sociedad. Hoy, se estima que en Colombia estas cifras de TI pueden ser mayores o estar en aumento -a pesar del período de crecimiento económico mencionado anteriormente y de la reducción del TI hasta el 2007- si se tiene en cuenta que, según el censo de 2005, existen unos 15.184.331 de personas menores de 18 años (37 \% de la población), de los cuales 4.965.624 (12\%) son menores de 5 años (DANE, 2007). De ellos, un 38.9 \% estaría en la 
línea de pobreza y un $17.5 \%$ en situación de miseria, y el $12 \%$ de los menores de 5 años presentaría desnutrición crónica o retardo en el crecimiento como consecuencia de la marginalidad y la pobreza (Consultoría para los Derechos Humanos y el Desplazamiento [CODHES], 2009).

Frente a esta problemática, se ha venido desarrollando una serie de acciones a nivel mundial, así como en Colombia, donde se encuentran estudios y planes importantes que brindaron información sobre el maltrato y la explotación laboral infantil como fenómeno social (Comité Interinstitucional Nacional para la Erradicación del Trabajo Infantil, 2003; Departamento Administrativo Nacional de Estadística [DANE], 2001; Díaz, 2004; Flórez \& Méndez, 1998, 1999; Guáqueta, Virgüez, Serrato \& Torrado, 2001; OIT-IPEC, 2005; OIT-Colombia 2003, 2006; Programa Interdisciplinario de Apoyo a la Comunidad [PRIAC], 2003; Rey, 2004). El enfoque teórico que lidera la mayor parte de estos estudios es el abolicionista, el cual considera la imperiosa necesidad de erradicar toda manifestación de TI (p. ej., OIT-IPEC). Frente a ella, se ubican otros estudios con una mirada del TI más centrada en cada niño como sujeto, en el interior de una sociedad incluyente y abierta, donde se requiere que sea el niño quien decida y asuma la manera en que puede relacionarse y representarse una actividad laboral. Esto se refleja en los niños considerados del grupo de los NAT (Niños y Adolescentes Trabajadores), representados sobre todo en Bolivia, Perú y Ecuador (Morsolin, 2007).

Entre los primeros estudios que despertaron el interés sobre esta situación en Colombia, se encuentran los realizados con $\mathrm{NNJ}$ que trabajaban en la industria del carbón y las flores, y a pesar de haberse elaborado una política pública en Colombia sobre esta materia, no se tiene mayor conocimiento sobre qué tan efectivos y sostenibles son sus resultados (Ordóñez \& Álvarez, 2006). Gracias a esta política se han materializado alianzas entre organizaciones del Estado y algunas ONG, y en el año 2009 se realizó la consolidación del Comité para la Erradicación del Trabajo Infantil (COETI), conformado por cooperantes internacionales que desarrollan proyectos de inversión en Colombia, como apoyo a la Estrategia Nacional de Erradicación del Trabajo Infantil y Protección al Joven trabajador 2008-2015. De esta alianza hacen parte la OIT a través de su proyecto IPEC, Visión Mundial Internacional, Save the Children, Fundación Telefónica y el proyecto Edúcame primero Colombia; cada una de estas entidades aporta los recursos y la experiencia de su trabajo, para lograr fortalecer los Comités Locales de Erradicación del Trabajo Infantil y ejecutar proyectos que buscan retirar a los niños del trabajo.

\section{Algunas causas del trabajo infantil}

Aunque es arriesgado definir una o varias causas directas que generan el trabajo infantil, se puede observar que este se asocia fuertemente con la pobreza, y su solución a largo plazo radica en un crecimiento económico sostenido, conducente al progreso social, en particular a la mitigación de la pobreza y a la educación universal (OIT, 2005, Convenio 182 sobre Eliminación de las Peores Formas del Trabajo Infantil, el TI). África, Asia y América Latina son las regiones más afectadas por la pobreza, y en América Latina más de la mitad de los NNA son pobres. En Colombia, se ha aumentado la pobreza de manera desproporcionada desde principios de la década de los 90 . Entre 1990 y el 2002 , esta pasó de $55 \%$ al $66 \%$, y se sabe que más de 25 millones de personas obtienen ingresos apenas para cubrir las necesidades alimenticias. Sin embargo, no solo la pobreza es relevante para el ingreso o mantenimiento de los NNA en el trabajo, también lo es el modelo de desarrollo económico de los países y las orientaciones políticas de estos, caracterizados por una gran inequidad y segmentación social, que incrementan los problemas estructurales de la sociedad (OIT- IPEC, 2002).

De esta manera, las familias pobres estructurales y las familias empobrecidas ante la situación de ajuste económico son colocadas en condición de vulnerabilidad social, perdiendo su capacidad económica y cultural de contención y disminuyendo sus posibilidades reales de alcanzar niveles de vida dignos. En muchas de ellas los NNA realizan diferentes actividades asimilables a la idea de trabajo, como estrategia de supervivencia. 
Al lado de la pobreza, se observa una intersección de múltiples factores personales, económicos, sociales y culturales en las familias, que ayudan al surgimiento y permanencia del trabajo infantil. Entre ellos, se encuentran las creencias o representaciones sobre la infancia, sobre el trabajo y la educación de los niños, así como valores y costumbres que condicionan las expectativas culturales. Tal como se ha identificado en los programas de atención a la niñez víctima del TI, "muchas veces los niños y niñas son articulados al trabajo por sus familias, quienes consideran que es propio o deseable como parte de su proceso de formación. Esta situación se acentúa con la desesperanza aprendida frente a las posibilidades de movilidad social y con la percepción de la educación como inapropiada para la satisfacción de las necesidades socioeconómicas y expectativas laborales y vitales" (OIT-IPEC, 2004, p. 10).

Otros factores que aportan al surgimiento y refuerzo del TI son la baja calidad del sistema educativo y la educación de los padres. Muchas comunidades no poseen instalaciones escolares, pero, incluso donde hay escuelas, la educación es algo que muchas familias no pueden permitirse aunque sea "gratuita", debido a que los ingresos perdidos cuando el niño no trabaja se consideran irremplazables. A esto se agrega que la educación ofrecida es de baja calidad, y los padres, e incluso los niños, consideran que no responde a las condiciones y necesidades locales (OIT \& UIP, 2002).

Por otra parte, de acuerdo con Sandoval (2007), la situación del trabajo infantil evidencia que a mayor grado de escolaridad de los padres, mayor será su intención de privilegiar en sus hijos el rol de estudiante; pero, frente a una baja escolaridad de los padres, estos se pueden orientar más a un ingreso temprano de los niños al mundo laboral. A pesar de esto, en los países subdesarrollados pocos padres manifiestan su deseo de poner a sus hijos menores a trabajar, y solo recurren a esto cuando las circunstancias los obligan (Basu, 1998).

En otras situaciones, el ingreso de los niños y las niñas al trabajo se ve relacionado con el abuso del poder en los hogares. Una parte de los niños y niñas víctimas del TI manifiestan que han sido objeto del maltrato físico por parte de sus padres o cuidadores, para obligarlos a trabajar en condiciones de alto riesgo, atentando contra su salud mental y física. Los niños y niñas se muestran sumisos ante estas manifestaciones de poder, que los obligan a doblegarse a las órdenes de la autoridad (Kempe $\&$ Kempe, 1978 citados en Rey, 2004). De acuerdo a la dinámica de poder que se da dentro de toda familia, los miembros más vulnerables quedan a merced de las leyes y normas que allí se fijan, por lo cual los niños y niñas son víctimas del control de los adultos, lo que propiciaría la explotación a través del TI. Como se puede observar, la condición de vulnerabilidad de la población infantil la convierte en presa fácil de diferentes delitos, lo que puede generar consecuencias nefastas que influirán en sus vivencias de adultos.

\section{Algunas consecuencias del trabajo infantil}

Como menciona la OIT, "esta población que proviene generalmente de los sectores económicos y sociales en desventaja, continúa reproduciendo su pobreza mediante la vinculación a trabajos poco calificados y mal remunerados en ocupaciones que siguen perpetuando su rezago en términos del tipo de procesos productivos que los caracterizan" (OIT-IPEC, 2004, p. 9). Los trabajos que realizan los niños, con frecuencia se desarrollan bajo relaciones de explotación, que llevan implícitas situaciones de discriminación, subordinación y sometimiento, que afectan el proceso de construcción de una identidad sana. Esta situación vulnera sus derechos a vivir dignamente y además les limita o anula sus derechos a la educación, a la recreación, al tiempo libre y a la posibilidad de socializar en espacios saludables.

Según los informes de la OIT en Colombia (OIT-IPEC, 2005), algunas posibles consecuencias de este flagelo, ocasionadas por accidentes laborales, violencia física, verbal, abuso sexual y discriminación, entre otros motivos, se observan en problemas de desnutrición, déficit de crecimiento, daños en su integridad física, baja autoestima, estrés, ansiedad y depresión, lo cual indica que pueden reflejar una amplia variedad de perjuicios en lo 
físico, lo mental, lo social y en su comportamiento moral. Además, "el trabajo en estas condiciones los condena a aceptar y 'naturalizar' una sociedad de ricos y pobres, capaces e incapaces, mejores y peores" (Lelièvre \& Ortiz, 2001 citados en Asociación Cristiana de Jóvenes de Bogotá [ACJB], 2005, p. 10), lo cual no hace sino dar una voz de alerta con respecto al éxito de su recuperación, y en la medida que los NNA pasen más tiempo bajo estas condiciones degradantes más difícil será que puedan gozar de una vida digna.

A nivel educativo se observa el bajo rendimiento escolar, o la interrupción o abandono escolar prematuro, ya que los NNA pierden la posibilidad de desarrollar sus potencialidades en un ambiente sano, sobre todo por tener que asumir responsabilidades laborales que no les permiten ingresar o permanecer en la escuela, y aquellos que tratan de mantenerse vinculados al sistema tienen un rendimiento académico bajo o nulo, que refuerza más la idea de deserción o son expulsados por del sistema por su bajo rendimiento o por dificultades en su comportamiento (ACJB, 2005).

Según Kempe y Kempe (1978 citados en Rey, 2004) los niños víctimas de maltrato, muestran dos patrones comportamentales: por un lado, estos niños y niñas pueden mostrarse complacientes, atentos e incluso ansiosos por agradar a los adultos y evitar cualquier castigo; por otro lado, pueden mostrarse provocadores y agresivos, probablemente porque de esta manera han aprendido a evitar los malos tratos físicos. Otro estudio realizado por IsonZintilini y Morelato-Giménez (2008), encontró que los niños víctimas de maltrato se diferencian de los niños con comportamientos delictivos, en la medida en que los niños maltratados presentaban más dificultades en las habilidades cognitivas de identificación de problemas, generación de alternativas de solución y anticipación de consecuencias; detectaban la emoción "miedo" en mayor proporción que los niños con conductas desviadas y, en la mayoría de los casos, no demostraban comportamientos de oposición por temor a castigos.

Frente a estos datos generales, presentados por organismos que realizan una labor social con esta población, se observa que no hay muchos datos actualizados sobre las características familiares de los niños trabajadores y en riesgo de serlo. Por esta razón, esta investigación intenta dar respuesta a la siguiente pregunta: ¿Cuáles son los factores familiares y sociales de alto riesgo asociados al trabajo infantil en niños, niñas y adolescentes entre 6 y 17 años de edad que participaron en el Proyecto "Edúcame primero, Colombia" en el Caribe colombiano?

\section{Método}

\section{Participantes}

La población estuvo constituida por todos los NNJ que participaron en el proyecto "Edúcame primero, Colombia” durante el año 2008. Este proyecto es producto del consorcio entre PARTNERS of the AMERICAS, Cinde, DevTech y Mercy Corps, con el apoyo del Ministerio de Trabajo de los Estados Unidos, en el cual la Universidad del Norte de Barranquilla, es uno de los socios regionales en la Costa Caribe. Los sujetos fueron $835 \mathrm{NNJ}$ entre 6 y 17 años $(M=10.6$ años y $D E=2.2)$ de las ciudades de Barranquilla 322 (38.6 \%), Santa Marta 224 (26.8 \%) y Cartagena 289 (34.6 \%). De ellos, 439 (52.6 \%) estaban en alto riesgo de trabajar y 396 (47.4\%) trabajaban o lo habían hecho en algún momento. La distribución en cuanto al género fue de 464 de género masculino (55.6\%) y 371 de género femenino (44.4\%). Su estrato socioeconómico se distribuyó en un $4.6 \%$ en el estrato 0, un $89 \%$ en el estrato 1 y un $6.5 \%$ entre los estratos 2 y 3 .

\section{Instrumento}

Se utilizó el "Formato de Inscripción de los Niños, Niñas y Adolescentes (NNA)" diseñado por el equipo de DevTech y la Fundación Centro Internacional de Educación y Desarrollo Humano (CINDE). El cuestionario se encuentra conformado por 43 preguntas con respuestas cerradas, múltiples o abiertas, sobre las características personales (nombre, barrio, etc.), familiares (si vive con los dos progenitores, si ambos padres viven, qué trabajo realizan, etc.), educativas (si se encuentra estudiando o no, grado de escolaridad, rendimiento en las materias básicas, 
etc.), y condición laboral actual de los niños (si se encuentra o no realizado alguna actividad laboral, cuántas horas trabaja a la semana, dónde trabaja, recibe algún pago por ese trabajo, etc.).

\section{Procedimiento}

A los NNJ y a uno de sus familiares se les entrevistó durante una hora aproximadamente en el momento en que estaban iniciando las actividades del Programa "Edúcame Primero, Colombia". Se realizaron visitas domiciliarias para controlar la consistencia de la información y luego se digitaron y procesaron los cuestionarios con el programa PASW Statistics 18.

\section{Resultados}

Características sociodemográficas de los niños y niñas víctimas (trabajadores) $y$ en riesgo (no trabajadores) de explotación laboral infantil

La investigación identificó que el 40.1 \% (335 casos) de la muestra estudiada es víctima de la explotación laboral infantil y un 59.8 \% (500 casos) se encontró en riesgo. De las tres ciudades participantes, Cartagena presentó mayor población de NNA víctimas de explotación laboral infantil o TI (66 \%). El análisis realizado sobre la distribución de acuerdo a la edad y la condición de víctima o en riesgo de

TABLA 1

Escolaridad de niños y niñas víctimas de TI por rango de edad (niños y niñas trabajadores)

\begin{tabular}{|c|c|c|c|c|c|c|c|c|c|c|}
\hline \multirow{3}{*}{ Escolaridad } & \multicolumn{8}{|c|}{ Rangos de edad } & \multirow{2}{*}{\multicolumn{2}{|c|}{ Total }} \\
\hline & \multicolumn{2}{|c|}{$\begin{array}{c}\text { De } 6 \text { a } 9 \\
\text { años }\end{array}$} & \multicolumn{2}{|c|}{$\begin{array}{c}\text { De } 10 \text { a } 12 \\
\text { años }\end{array}$} & \multicolumn{2}{|c|}{$\begin{array}{c}\text { De } 13 \text { a } 14 \\
\text { años }\end{array}$} & \multicolumn{2}{|c|}{$\begin{array}{c}\text { De } 15 \text { a } 17 \\
\text { años }\end{array}$} & & \\
\hline & Fr. & $\%$ & Fr. & $\%$ & Fr. & $\%$ & Fr. & $\%$ & Fr. & $\%$ \\
\hline No escolarizado & 4 & 1.2 & 2 & 0.6 & 0 & 0 & 0 & 0 & 6 & 1.8 \\
\hline Primaria & 66 & 19.7 & 118 & 35.2 & 37 & 11 & 13 & 3.9 & 234 & 69.9 \\
\hline Secundaria & 1 & 0.3 & 48 & 14.3 & 36 & 10.7 & 10 & 3 & 95 & 28.4 \\
\hline Total & 71 & 21.2 & 168 & 50.1 & 73 & 21.8 & 23 & 6.9 & 335 & 100 \\
\hline
\end{tabular}

Fuente: elaboración propia.

TABLA 2

Escolaridad de niños y niñas en riesgo de TI por rango de edad (niños y niñas trabajadores)

\begin{tabular}{|c|c|c|c|c|c|c|c|c|c|c|}
\hline \multirow{3}{*}{ Escolaridad } & \multicolumn{8}{|c|}{ Rangos de edad } & \multirow{2}{*}{\multicolumn{2}{|c|}{ Total }} \\
\hline & \multicolumn{2}{|c|}{$\begin{array}{c}\text { De } 6 \text { a } 9 \\
\text { años }\end{array}$} & \multicolumn{2}{|c|}{$\begin{array}{c}\text { De } 10 \text { a } 12 \\
\text { años }\end{array}$} & \multicolumn{2}{|c|}{$\begin{array}{c}\text { De } 13 \text { a } 14 \\
\text { años }\end{array}$} & \multicolumn{2}{|c|}{$\begin{array}{c}\text { De } 15 \text { a } 17 \\
\text { años }\end{array}$} & & \\
\hline & Fr. & $\%$ & Fr. & $\%$ & Fr. & $\%$ & Fr. & $\%$ & Fr. & $\%$ \\
\hline No escolarizado & 3 & 0.6 & 0 & 0 & 0 & 0 & 1 & 0.2 & 4 & 0.8 \\
\hline Primaria & 203 & 40.6 & 171 & 34.2 & 38 & 7.6 & 8 & 1.6 & 420 & 84.0 \\
\hline Secundaria & 3 & 0.6 & 36 & 7.2 & 31 & 6.2 & 6 & 1.2 & 76 & 15.2 \\
\hline Total & 209 & 41.8 & 207 & 41.4 & 69 & 13.8 & 15 & 3 & 500 & 100 \\
\hline
\end{tabular}

Fuente: elaboración propia. 
TABLA 3

Ocupación de la madre en familias con niños y niñas víctimas de TI (niños y niñas trabajadores)

\begin{tabular}{|c|c|c|c|c|c|c|c|c|c|c|c|c|c|c|c|c|c|c|}
\hline \multirow{3}{*}{ Ciudad } & \multicolumn{16}{|c|}{ Ocupación de la madre } & \multirow{2}{*}{\multicolumn{2}{|c|}{ Total }} \\
\hline & \multicolumn{2}{|c|}{$\begin{array}{c}\text { Trabajó (o } \\
\text { trabaja o } \\
\text { estudia) }\end{array}$} & \multicolumn{2}{|c|}{$\begin{array}{c}\text { No } \\
\text { trabajó } \\
\text { pero } \\
\text { tenía } \\
\text { trabajo }\end{array}$} & \multicolumn{2}{|c|}{$\begin{array}{c}\text { Buscó } \\
\text { traba- } \\
\text { jo pero } \\
\text { había } \\
\text { trabajado } \\
\text { antes }\end{array}$} & \multicolumn{2}{|c|}{$\begin{array}{l}\text { Buscó tra- } \\
\text { bajo por } \\
\text { primera } \\
\text { vez }\end{array}$} & \multicolumn{2}{|c|}{$\begin{array}{c}\text { Estudió y } \\
\text { no trabajó } \\
\text { ni buscó } \\
\text { trabajo }\end{array}$} & \multicolumn{2}{|c|}{$\begin{array}{l}\text { Realizó } \\
\text { oficios del } \\
\text { hogar y } \\
\text { no trabajó } \\
\text { ni buscó } \\
\text { trabajo }\end{array}$} & \multicolumn{2}{|c|}{$\begin{array}{c}\text { Estuvo } \\
\text { en otra } \\
\text { situación }\end{array}$} & \multicolumn{2}{|c|}{$\begin{array}{l}\text { Estuvo in- } \\
\text { capacitado } \\
\text { permanente- } \\
\text { mente para } \\
\text { trabajar }\end{array}$} & & \\
\hline & Fr. & $\%$ & Fr. & $\%$ & Fr. & $\%$ & Fr. & $\%$ & Fr. & $\%$ & Fr. & $\%$ & Fr. & $\%$ & Fr. & $\%$ & Fr. & $\%$ \\
\hline B/quilla & 38 & 11.3 & 4 & 1.2 & 0 & 0 & 0 & 0 & 0 & 0 & 50 & 14.9 & 3 & 0.9 & 0 & 0 & 95 & 28.4 \\
\hline C/gena & 110 & 32.8 & 12 & 3.6 & 8 & 2.4 & 1 & 0.3 & 1 & 0.3 & 84 & 25.1 & 5 & 1.5 & 0 & 0 & 221 & 66 \\
\hline S/Marta & 8 & 2.4 & 2 & 0.6 & 1 & 0.3 & 0 & 0 & 0 & 0 & 7 & 2.1 & 0 & 0 & 1 & 0.3 & 19 & 5.7 \\
\hline Total & 156 & 46.6 & 18 & 5.4 & 9 & 2.7 & 1 & 0.3 & 1 & 0.3 & 141 & 42.1 & 8 & 2.4 & 1 & 0.3 & 335 & 100 \\
\hline
\end{tabular}

Fuente: elaboración propia.

TABLA 4

Ocupación de la madre en familias con niños y niñas en riesgo de TI (niños y niñas no trabajadores)

\begin{tabular}{|c|c|c|c|c|c|c|c|c|c|c|c|c|c|c|c|c|}
\hline \multirow{3}{*}{ Ciudad } & \multicolumn{14}{|c|}{ Ocupación de la madre } & \multirow{2}{*}{\multicolumn{2}{|c|}{ Total }} \\
\hline & \multicolumn{2}{|c|}{ Trabajó } & \multicolumn{2}{|c|}{$\begin{array}{l}\text { No tra- } \\
\text { bajó pero } \\
\text { tenía } \\
\text { trabajo }\end{array}$} & \multicolumn{2}{|c|}{$\begin{array}{c}\text { Buscó } \\
\text { trabajo } \\
\text { pero había } \\
\text { trabajado } \\
\text { antes }\end{array}$} & \multicolumn{2}{|c|}{$\begin{array}{l}\text { Buscó tra- } \\
\text { bajo por } \\
\text { primera } \\
\text { vez }\end{array}$} & \multicolumn{2}{|c|}{$\begin{array}{c}\text { Estudió y } \\
\text { no trabajó } \\
\text { ni buscó } \\
\text { trabajo }\end{array}$} & \multicolumn{2}{|c|}{$\begin{array}{l}\text { Realizó } \\
\text { oficios del } \\
\text { hogar, } \\
\text { no trabajó } \\
\text { ni buscó } \\
\text { trabajo }\end{array}$} & \multicolumn{2}{|c|}{$\begin{array}{c}\text { Estuvo en } \\
\text { otra situa- } \\
\text { ción }\end{array}$} & & \\
\hline & Fr. & $\%$ & Fr. & $\%$ & Fr. & $\%$ & Fr. & $\%$ & Fr. & $\%$ & Fr. & $\%$ & Fr. & $\%$ & Fr. & $\%$ \\
\hline Barranquilla & 83 & 16.6 & 13 & 2.6 & 0 & 0 & 0 & 0 & 0 & 0 & 126 & 25.2 & 5 & 1.0 & 227 & 45.4 \\
\hline Cartagena & 29 & 5.8 & 12 & 2.4 & 1 & 0.2 & 0 & 0 & 0 & 0 & 26 & 5.2 & 0 & 0 & 68 & 13.6 \\
\hline Santa Marta & 74 & 14.8 & 8 & 1 & 9 & 1.8 & 4 & 0.8 & 4 & 0.8 & 103 & 20.6 & 3 & 0.6 & 205 & 41 \\
\hline Total & 186 & 37.2 & 33 & 6.6 & 10 & 2 & 4 & 0.8 & 4 & 0.8 & 255 & 51 & 8 & 1.6 & 500 & 100 \\
\hline
\end{tabular}

Fuente: elaboración propia.

explotación laboral infantil indica que esta ciudad presenta mayor porcentaje de niños y niñas entre los 10 y 12 años víctimas de TI (38.5 \%) mientras que la mayor población de niños en riesgo entre los 6 y 9 años de edad la tienen Barranquilla (19.2\%) y Santa Marta (18.4\%), y entre 10 y 12 años de edad $(17.8 \%)$ y $(16.8 \%)$, respectivamente.

Como se explicó, el TI tiene efectos negativos sobre la asistencia escolar de los niños y niñas que lo sufren y aumenta su nivel de extra-edad en el sistema educativo, lo que se pudo observar en algu- nos casos de la muestra estudiada. De acuerdo a la escolaridad, se encontró que un 69.9 \% de las víctimas de TI se encuentra en primaria o finalizando este nivel, mientras un $84 \%$ de los niños y niñas en riesgo se encuentran cursando este grado (Tablas 1 y 2). Datos complementarios a este análisis muestran que los NNJ ejercen algún tipo de trabajo en la jornada alterna de sus estudios, es decir, si estudian en la mañana trabajan por la tarde y viceversa, y en algunos casos lo hacen los fines de semana. 
TABLA 5

Ocupación del padre en familias con niños y niñas en riesgo de TI (niños y niñas no trabajadores)

\begin{tabular}{|c|c|c|c|c|c|c|c|c|c|c|c|c|c|c|c|c|c|c|}
\hline \multirow{3}{*}{ Ciudad } & \multicolumn{16}{|c|}{ Ocupación del padre } & \multirow{2}{*}{\multicolumn{2}{|c|}{ Total }} \\
\hline & \multicolumn{2}{|c|}{ Trabajó } & \multicolumn{2}{|c|}{$\begin{array}{l}\text { No } \\
\text { trabajó } \\
\text { pero tenía } \\
\text { trabajo }\end{array}$} & \multicolumn{2}{|c|}{$\begin{array}{c}\text { Buscó } \\
\text { trabajo } \\
\text { pero } \\
\text { había } \\
\text { trabajado } \\
\text { antes }\end{array}$} & \multicolumn{2}{|c|}{$\begin{array}{c}\text { Buscó } \\
\text { trabajo } \\
\text { por } \\
\text { primera } \\
\text { vez }\end{array}$} & \multicolumn{2}{|c|}{$\begin{array}{c}\text { Estudió } \\
\text { y no } \\
\text { trabajó, } \\
\text { ni buscó } \\
\text { trabajo }\end{array}$} & \multicolumn{2}{|c|}{$\begin{array}{c}\text { Realizó } \\
\text { oficios } \\
\text { del hogar } \\
\text { y no } \\
\text { trabajó, } \\
\text { ni buscó } \\
\text { trabajo }\end{array}$} & \multicolumn{2}{|c|}{$\begin{array}{c}\text { Estuvo } \\
\text { en otra } \\
\text { situación }\end{array}$} & \multicolumn{2}{|c|}{$\begin{array}{c}\text { Tuvo } \\
\text { incapacidad } \\
\text { permanente } \\
\text { para trabajar }\end{array}$} & & \\
\hline & Fr. & $\%$ & Fr. & $\%$ & Fr. & $\%$ & Fr. & $\%$ & Fr. & $\%$ & Fr. & $\%$ & Fr. & $\%$ & Fr. & $\%$ & Fr. & $\%$ \\
\hline B/quilla & 147 & 29.4 & 63 & 12.6 & 0 & 0 & 0 & 0 & 0 & 0 & 0 & 0 & 17 & 3.4 & 0 & 0 & 227 & 45.4 \\
\hline Cartagena & 32 & 6.4 & 33 & 6.6 & 0 & 0 & 0 & 0 & 1 & 0.2 & 0 & 0 & 2 & 0.4 & 0 & 0 & 68 & 13.6 \\
\hline S/Marta & 131 & 26.2 & 46 & 9.2 & 5 & 1 & 1 & 0.2 & 1 & 0.2 & 3 & 0.6 & 17 & 3.4 & 1 & 0.2 & 205 & 41 \\
\hline Total & 310 & 62 & 142 & 28.4 & 5 & 1 & 1 & 0.2 & 2 & 0.4 & 3 & 0.6 & 36 & 7.2 & 1 & 0.2 & 500 & 100 \\
\hline
\end{tabular}

Fuente: elaboración propia.

TABLA 6

Ocupación del padre en familias con niños y niñas víctimas de TI (niños y niñas trabajadores)

\begin{tabular}{|c|c|c|c|c|c|c|c|c|c|c|c|c|c|c|}
\hline \multirow{3}{*}{ Ciudad } & \multicolumn{12}{|c|}{ Ocupación del padre } & \multirow{2}{*}{\multicolumn{2}{|c|}{ Total }} \\
\hline & \multicolumn{2}{|c|}{ Trabajó } & \multicolumn{2}{|c|}{$\begin{array}{c}\text { No trabajó } \\
\text { pero tenía } \\
\text { trabajo }\end{array}$} & \multicolumn{2}{|c|}{$\begin{array}{c}\text { Buscó } \\
\text { trabajo } \\
\text { pero había } \\
\text { trabajado } \\
\text { antes } \\
\end{array}$} & \multicolumn{2}{|c|}{$\begin{array}{c}\text { Buscó } \\
\text { trabajo por } \\
\text { primera vez }\end{array}$} & \multicolumn{2}{|c|}{$\begin{array}{c}\text { Vivió de } \\
\text { jubilación o } \\
\text { renta y no } \\
\text { trabajó ni } \\
\text { buscó trabajo } \\
\end{array}$} & \multicolumn{2}{|c|}{$\begin{array}{c}\text { Estuvo } \\
\text { en otra } \\
\text { situación }\end{array}$} & & \\
\hline & Fr. & $\%$ & Fr. & $\%$ & Fr. & $\%$ & Fr. & $\%$ & Fr. & $\%$ & Fr. & $\%$ & Fr. & $\%$ \\
\hline Barranquilla & 68 & 20.3 & 18 & 5.4 & 0 & 0 & 0 & 0 & 1 & 0.3 & 8 & 2.4 & 95 & 28.4 \\
\hline Cartagena & 122 & 36.4 & 66 & 19.7 & 8 & 2.4 & 1 & 0.3 & 0 & 0 & 24 & 7.2 & 221 & 66 \\
\hline Santa Marta & 11 & 3.3 & 7 & 2.1 & 0 & 0 & 0 & 0 & 0 & 0 & 1 & 0.3 & 19 & 5.7 \\
\hline Total & 201 & 60 & 91 & 27.2 & 8 & 2.4 & 1 & 0.3 & 1 & 0.3 & 33 & 9.9 & 335 & 100 \\
\hline
\end{tabular}

Fuente: elaboración propia.

Características familiares de los niños y niñas víctimas y en riesgo de explotación laboral infantil o TI

En las familias de la muestra estudiada, es recurrente que los jefes de hogar se dediquen a trabajos informales y eventuales. No se encontraron diferencias significativas entre los porcentajes de madres que no trabajan, tanto de niños y niñas víctimas como de los que están en riesgo de TI, para las ciudades de Barranquilla (27.8 \% madres de niños y niñas en riesgo) y Cartagena (28.7 \% madres de niños y niñas víctimas). Ambos porcentajes corresponden a la suma de los valores de "no trabajó pero tenía trabajo y realizó oficios en el hogar" (Tablas 7 y 8).

Con respecto a las madres que trabajan, lo hacen en mayor porcentaje (32.8 \%) las madres de los niños y niñas víctimas (trabajadores) de explotación laboral infantil o TI, pertenecientes a la ciudad de Cartagena, donde la proporción de estos niños y niñas es mayor con respecto a las demás ciudades (Tabla 7). Tanto en Barranquilla como en Santa Marta y Cartagena, se hallaron casos de madres en otro tipo de situación, como fallecimiento (4\%), 
incapacidad física (0.3\%), estudios (1.1\%), no trabajaron pero tenían trabajo (12 \%) o lo habían hecho alguna vez (4.7\%).

Con respecto a los padres, se identifica que los que trabajan se encuentran en proporciones diferentes para cada una de las tres ciudades. En Barranquilla, los padres de los niños y niñas en riesgo de explotación laboral infantil se encuentran trabajando (29.4\%), en Cartagena lo hacen en un $6.4 \%$ y en Santa Marta un 26.2 \% (Tablas 9 y 10).

En las familias donde los niños y niñas son víctimas de la explotación laboral infantil, los padres trabajan. En el caso de Cartagena, un 36.4 \% de los padres de estos niños y niñas se encuentran laborando. En Barranquilla, el 20.3 \% lo hace y en Santa Marta un 3.3 \% (Tabla 6).

Si se compara en Cartagena el porcentaje de padres que trabajan (36.4\%) con el de los que no se encuentran laborando, en la población de niños y niñas víctimas de TI, son más los padres que no trabajan que los que sí lo hacen para un total de 39.8 \% (suma de porcentajes de "no trabajo pero tenía trabajo, buscó trabajo pero había trabajado antes, buscó trabajo por primera vez, vivió de jubilación o estuvo en otra situación como fallecido o discapacitado"). Sin embargo, para la muestra, Cartagena presenta un alto porcentaje de niños y niñas cuyos padres han fallecido (7.2 \%), razón por la que a estos niños y niñas les ha tocado ir a trabajar, con el fin de ayudar a los gastos del hogar (de acuerdo a la gráfica corresponde al ítem "estuvo en otra situación").

Con respecto a la escolaridad de los padres y las madres de los niños y niñas víctimas y en riesgo de TI, se encontró que en las familias de los niños y niñas en riesgo, los estudios escolares del padre llegan hasta algún nivel de secundaria (31.2\%, Tabla 11), mientras que en las familias de niños y niñas víctimas de TI, los estudios escolares del padre alcanzan un porcentaje de $33.1 \%$ en el nivel primaria (Tabla 12). Se pudo observar que en estas familias los padres ejercen autoridad en distintas áreas de la dinámica familiar y deciden si sus hijos abandonan la escuela para ayudar con los gastos de la casa o si continúan estudiando. Sin embargo,

TABLA 7

Escolaridad del padre en familias con niños y niñas en riesgo de TI (niños y niñas no trabajadores)

\begin{tabular}{|c|c|c|c|c|c|c|c|c|c|c|}
\hline \multirow{3}{*}{ Ciudad } & \multicolumn{8}{|c|}{ Escolaridad del padre } & \multirow{2}{*}{\multicolumn{2}{|c|}{ Total }} \\
\hline & \multicolumn{2}{|c|}{ No escolarizado } & \multicolumn{2}{|c|}{ Primaria } & \multicolumn{2}{|c|}{ Secundaria } & \multicolumn{2}{|c|}{ No aplica } & & \\
\hline & Fr. & $\%$ & Fr. & $\%$ & Fr. & $\%$ & Fr. & $\%$ & Fr. & $\%$ \\
\hline Barranquilla & 79 & 15.8 & 58 & 11.6 & 75 & 15 & 15 & 3 & 227 & 45.4 \\
\hline Cartagena & 37 & 7.4 & 20 & 4 & 11 & 2.2 & 0 & 0 & 68 & 13.6 \\
\hline Santa Marta & 64 & 12.8 & 66 & 13.2 & 70 & 14 & 5 & 1.0 & 205 & 41 \\
\hline Total & 180 & 36 & 144 & 28.8 & 156 & 31.2 & 20 & 4.0 & 500 & 100 \\
\hline
\end{tabular}

Fuente: elaboración propia.

TABLA 8

Escolaridad del padre en familias con niños y niñas víctimas de TI (niños y niñas trabajadores)

\begin{tabular}{|c|c|c|c|c|c|c|c|c|c|c|}
\hline \multirow{3}{*}{ Ciudad } & \multicolumn{8}{|c|}{ Escolaridad del padre } & \multirow{2}{*}{\multicolumn{2}{|c|}{ Total }} \\
\hline & \multicolumn{2}{|c|}{ No escolarizado } & \multicolumn{2}{|c|}{ Primaria } & \multicolumn{2}{|c|}{ Secundaria } & \multicolumn{2}{|c|}{ No aplica } & & \\
\hline & Fr. & $\%$ & Fr. & $\%$ & Fr. & $\%$ & Fr. & $\%$ & Fr. & $\%$ \\
\hline Barranquilla & 29 & 8.7 & 42 & 12.5 & 20 & 6 & 4 & 1.2 & 95 & 28.4 \\
\hline Cartagena & 92 & 27.5 & 59 & 17.6 & 62 & 18.5 & 8 & 2.4 & 221 & 66 \\
\hline Santa Marta & 7 & 2.1 & 10 & 3 & 1 & 0.3 & 1 & 0.3 & 19 & 5.7 \\
\hline Total & 128 & 38.2 & 111 & 33.1 & 83 & 24.8 & 13 & 3.9 & 335 & 100 \\
\hline
\end{tabular}

Fuente: elaboración propia. 
TABLA 9

Escolaridad de la madre en familias con niños y niñas en riesgo de TI (niños y niñas no trabajadores)

\begin{tabular}{|c|c|c|c|c|c|c|c|c|c|c|}
\hline \multirow{3}{*}{ Ciudad } & \multicolumn{8}{|c|}{ Escolaridad de la madre } & \multirow{2}{*}{\multicolumn{2}{|c|}{ Total }} \\
\hline & \multicolumn{2}{|c|}{ No escolarizado } & \multicolumn{2}{|c|}{ Primaria } & \multicolumn{2}{|c|}{ Secundaria } & \multicolumn{2}{|c|}{ No aplica } & & \\
\hline & Fr. & $\%$ & Fr. & $\%$ & Fr. & $\%$ & Fr. & $\%$ & Fr. & $\%$ \\
\hline Barranquilla & 21 & 4.2 & 88 & 17.6 & 114 & 22.8 & 4 & 0.8 & 227 & 45.4 \\
\hline Cartagena & 14 & 2.8 & 41 & 8.2 & 13 & 2.6 & 0 & 0 & 68 & 13.6 \\
\hline Santa Marta & 19 & 3.8 & 108 & 21.6 & 77 & 15.4 & 1 & 0.2 & 205 & 41 \\
\hline Total & 54 & 10.8 & 237 & 47.4 & 204 & 40.8 & 5 & 1.0 & 500 & 100 \\
\hline
\end{tabular}

Fuente: elaboración propia.

TABLA 10

Escolaridad de la madre en familias con niños y niñas víctimas de TI (niños y niñas trabajadores)

\begin{tabular}{|c|c|c|c|c|c|c|c|c|c|c|}
\hline \multirow{3}{*}{ Ciudad } & \multicolumn{8}{|c|}{ Escolaridad de la madre } & \multirow{2}{*}{\multicolumn{2}{|c|}{ Total }} \\
\hline & \multicolumn{2}{|c|}{ No escolarizado } & \multicolumn{2}{|c|}{ Primaria } & \multicolumn{2}{|c|}{ Secundaria } & \multicolumn{2}{|c|}{ No aplica } & & \\
\hline & Fr. & $\%$ & Fr. & $\%$ & Fr. & $\%$ & Fr. & $\%$ & Fr. & $\%$ \\
\hline Barranquilla & 27 & 8.1 & 48 & 14.3 & 20 & 6 & 0 & 0 & 95 & 28.4 \\
\hline Cartagena & 31 & 9.3 & 91 & 27.2 & 98 & 29.3 & 1 & 0.3 & 221 & 66 \\
\hline Santa Marta & 4 & 1.2 & 10 & 3 & 5 & 1.5 & 0 & 0 & 19 & 5.7 \\
\hline Total & 62 & 18.5 & 149 & 44.5 & 123 & 36.7 & 1 & 0.3 & 335 & 100 \\
\hline
\end{tabular}

Fuente: elaboración propia.

TABLA 11

Relación con los progenitores en familias de niños y niñas en riesgo de TI (niños y niñas no trabajadores)

\begin{tabular}{|c|c|c|c|c|c|c|c|c|c|c|}
\hline \multirow{3}{*}{ Ciudad } & \multicolumn{8}{|c|}{ Relación con sus progenitores (convive) } & \multirow{2}{*}{\multicolumn{2}{|c|}{ Total }} \\
\hline & \multicolumn{2}{|c|}{$\begin{array}{c}\text { Con los } \\
\text { dos progenitores }\end{array}$} & \multicolumn{2}{|c|}{$\begin{array}{c}\text { Únicamente } \\
\text { con } \\
\text { la madre }\end{array}$} & \multicolumn{2}{|c|}{$\begin{array}{l}\text { Únicamente } \\
\text { con el padre }\end{array}$} & \multicolumn{2}{|c|}{ Con otras personas } & & \\
\hline & Fr. & $\%$ & Fr. & $\%$ & Fr. & $\%$ & Fr. & $\%$ & Fr. & $\%$ \\
\hline Barranquilla & 125 & 25.0 & 80 & 16 & 9 & 1.8 & 13 & 2.6 & 227 & 45.4 \\
\hline Cartagena & 22 & 4.4 & 17 & 3.4 & 5 & 1 & 24 & 4.8 & 68 & 13.6 \\
\hline Santa Marta & 122 & 24.4 & 57 & 11.4 & 8 & 1.6 & 18 & 3.6 & 205 & 41 \\
\hline Total & 269 & 53.8 & 154 & 30.8 & 22 & 4.4 & 55 & 11 & 500 & 100 \\
\hline
\end{tabular}

Fuente: elaboración propia.

no siempre continuar en la escuela significa que los niños y niñas han dejado de trabajar.

En lo referente a las madres de los niños y niñas en riesgo de $\mathrm{TI}$, se encuentra que han llegado hasta primaria (47.4\%) y secundaria (40.8\%) (Tabla 13$)$.

Sin embargo, en los niños y niñas que han sido víctimas de TI, la diferencia es significativa en el porcentaje con respecto a las madres que han alcanzado el nivel primaria (44.5\%) y las madres que han llegado a cursar algún nivel de secundaria (36.7 \%) (Tabla 14).

Tal como se ha mencionado, la escolaridad de los progenitores es una variable que juega un papel muy importante para el análisis de las características de la población estudiada. En las familias donde viven los dos progenitores o uno de ellos (el caso 
TABLA 12

Relación con los progenitores en familias de niños y niñas víctimas de TI (niños y niñas no trabajadores)

\begin{tabular}{|c|c|c|c|c|c|c|c|c|c|c|}
\hline \multirow{3}{*}{ Ciudad } & \multicolumn{8}{|c|}{ Relación con sus progenitores (convive) } & \multirow{2}{*}{\multicolumn{2}{|c|}{ Total }} \\
\hline & \multicolumn{2}{|c|}{$\begin{array}{c}\text { Con los } \\
\text { dos progenitores }\end{array}$} & \multicolumn{2}{|c|}{$\begin{array}{c}\text { Únicamente } \\
\text { con } \\
\text { la madre }\end{array}$} & \multicolumn{2}{|c|}{$\begin{array}{l}\text { Únicamente } \\
\text { con el padre }\end{array}$} & \multicolumn{2}{|c|}{$\begin{array}{l}\text { Con otras } \\
\text { personas }\end{array}$} & & \\
\hline & Fr. & $\%$ & Fr. & $\%$ & Fr. & $\%$ & Fr. & $\%$ & Fr. & $\%$ \\
\hline Barranquilla & 64 & 19.1 & 21 & 6.3 & 5 & 1.5 & 5 & 1.5 & 95 & 28.4 \\
\hline Cartagena & 99 & 29.6 & 76 & 22.7 & 9 & 2.7 & 37 & 11 & 221 & 66 \\
\hline Santa Marta & 10 & 3 & 9 & 2.7 & 0 & 0 & 0 & 0 & 19 & 5.7 \\
\hline Total & 173 & 51.6 & 106 & 31.6 & 14 & 4.2 & 42 & 12.5 & 335 & 100 \\
\hline
\end{tabular}

Fuente: elaboración propia.

más representativo es la madre) que hayan alcanzado a cursar primaria, se evidencia mayor población de niños y niñas víctimas de TI, independiente de si estos últimos se encuentren estudiando o no. Se puede inferir que las creencias y las prácticas en torno a la educación en estas familias, puede ser un condicionante para que el TI se perpetúe, por la réplica de las conductas de los padres en los hijos o por la falta de recursos económicos en el hogar que facilita el ingreso en el trabajo de otros miembros de las familias, como los niños y niñas.

En lo que se refiere a la estructura familiar de la muestra estudiada, esta se caracteriza por mostrar, para ambos tipos de población $(53.8 \%$ no trabajadores y $51.6 \%$ trabajadores) en las tres ciudades, la presencia de ambos padres biológicos viviendo dentro del hogar (Tablas 11 y 12).

Un dato importante para analizar en la ciudad de Cartagena, es el porcentaje significativo de familias cuyos padres viven en el mismo hogar (29.6 \%) y, sin embargo, presentan niños y niñas víctimas de TI. De la misma manera, se da esta situación cuando viven únicamente con su madre (22.7\%) (Tabla 12). En estos últimos casos, el padre ha fallecido, al igual que cuando viven con otras personas $(11 \%)$ como abuelas, tíos u otros parientes.

Es importante analizar que en la historia familiar de estos niños, ambos progenitores empezaron sus actividades laborales a temprana edad (véanse Tablas 13, 14, 15 y 16).
Esto puede sugerir que la condición de trabajo del padre o de la madre antes de los 15 años, no es un detonante o una variable significativa para el ingreso de los niños y niñas al trabajo. De acuerdo a los estudios antes mencionados, el sistema de creencias y valores, así como el significado que la familia tenga sobre la educación, siguen siendo los factores más influyentes para que el TI aparezca, más allá de la situación económica que se viva dentro de los hogares.

\section{Discusión}

El estudio permitió observar que los niños se encontraron en mayor proporción dentro de las víctimas de TI que las niñas. Esto indica una situación, en la región Caribe colombiana, que resultaría importante analizar en posteriores estudios con mayor profundidad. Investigaciones realizadas en otras zonas del país, también mostraron dichas diferencias; ejemplo de esto es la investigación de Pinzón, Briceño, Gómez y Latorre (2003), quienes identificaron la existencia de una mayor población de niños que de niñas trabajando y una parte de estos niños se encontraba acompañada por un adulto, ayudándolos en las ventas. Estas diferencias poblacionales también pueden deberse a factores culturales y sociales del lugar donde se produce este fenómeno.

Los resultados de este estudio, que apuntan a la edad de vinculación al trabajo, muestran un predominio del inicio antes de los 12 años, y se identifica que sus padres también iniciaron actividad laboral a 
TABLA 13

Condición de trabajo de los padres antes de los 18 años (niños y niñas en riesgo de TI)

\begin{tabular}{|c|c|c|c|c|c|c|c|c|}
\hline \multirow{3}{*}{ Ciudad } & \multicolumn{6}{|c|}{ Trabajó cuando era niño - Padre } & \multirow{2}{*}{\multicolumn{2}{|c|}{ Total }} \\
\hline & \multicolumn{2}{|c|}{ Antes de los 15 años } & \multicolumn{2}{|c|}{ Entre los 16 y 18 años } & \multicolumn{2}{|c|}{ No trabajó } & & \\
\hline & Fr. & $\%$ & Fr. & $\%$ & Fr. & $\%$ & Fr. & $\%$ \\
\hline Barranquilla & 119 & 23.8 & 42 & 8.4 & 66 & 13.2 & 227 & 45.4 \\
\hline Cartagena & 38 & 7.6 & 12 & 2.4 & 18 & 3.6 & 68 & 13.6 \\
\hline Santa Marta & 110 & 22 & 36 & 7.2 & 59 & 11.8 & 205 & 41 \\
\hline Total & 267 & 53.4 & 90 & 18 & 143 & 28.6 & 500 & 100 \\
\hline
\end{tabular}

Fuente: elaboración propia.

TABLA 14

Condición de trabajo de los padres antes de los 18 años en familias de niños y niñas víctimas de TI

\begin{tabular}{|c|c|c|c|c|c|c|c|c|}
\hline \multirow{3}{*}{ Ciudad } & \multicolumn{6}{|c|}{ Trabajó cuando era niño - Padre } & \multirow{2}{*}{\multicolumn{2}{|c|}{ Total }} \\
\hline & \multicolumn{2}{|c|}{ Antes de los 15 años } & \multicolumn{2}{|c|}{ Entre los 16 y 18 años } & \multicolumn{2}{|c|}{ No trabajó } & & \\
\hline & Fr. & $\%$ & Fr. & $\%$ & Fr. & $\%$ & Fr. & $\%$ \\
\hline Barranquilla & 51 & 15.2 & 35 & 10.4 & 9 & 2.7 & 95 & 28.4 \\
\hline Cartagena & 130 & 38.8 & 39 & 11.6 & 52 & 15.5 & 221 & 66 \\
\hline Santa Marta & 10 & 3 & 3 & 0.9 & 6 & 1.8 & 19 & 5.7 \\
\hline Total & 191 & 57.0 & 77 & 23 & 67 & 20 & 335 & 100 \\
\hline
\end{tabular}

Fuente: elaboración propia.

TABLA 15

Condición de trabajo de las madres antes de los 18 años en familias de niños y niñas en riesgo de TI

\begin{tabular}{|c|c|c|c|c|c|c|c|c|}
\hline \multirow{3}{*}{ Ciudad } & \multicolumn{6}{|c|}{ Trabajó cuando era niña - Madre } & \multirow{2}{*}{\multicolumn{2}{|c|}{ Total }} \\
\hline & \multicolumn{2}{|c|}{ Antes de los 15 años } & \multicolumn{2}{|c|}{ Entre los 16 y 18 años } & \multicolumn{2}{|c|}{ No trabajó } & & \\
\hline & Fr. & $\%$ & Fr. & $\%$ & Fr. & $\%$ & Fr. & $\%$ \\
\hline Barranquilla & 87 & 17.4 & 26 & 5.2 & 114 & 22.8 & 227 & 45.4 \\
\hline Cartagena & 37 & 7.4 & 7 & 1.4 & 24 & 4.8 & 68 & 13.6 \\
\hline Santa Marta & 112 & 22.4 & 20 & 4 & 73 & 14.6 & 205 & 41 \\
\hline Total & 236 & 47.2 & 53 & 10.6 & 211 & 42.2 & 500 & 100 \\
\hline
\end{tabular}

Fuente: elaboración propia.

TABLA 16

Condición de trabajo de las madres antes de los 18 años en familias de niños y niñas víctimas de TI

\begin{tabular}{|c|c|c|c|c|c|c|c|c|}
\hline \multirow{3}{*}{ Ciudad } & \multicolumn{6}{|c|}{ Trabajo cuando era niña - Madre } & \multirow{2}{*}{\multicolumn{2}{|c|}{ Total }} \\
\hline & \multicolumn{2}{|c|}{ Antes de los 15 años } & \multicolumn{2}{|c|}{ Entre los 16 y 18 años } & \multicolumn{2}{|c|}{ No trabajó } & & \\
\hline & Fr. & $\%$ & Fr. & $\%$ & Fr. & $\%$ & Fr. & $\%$ \\
\hline Barranquilla & 52 & 15.5 & 14 & 4.2 & 29 & 8.7 & 95 & 28.4 \\
\hline Cartagena & 99 & 29.6 & 34 & 10.1 & 88 & 26.3 & 221 & 66 \\
\hline Santa Marta & 14 & 4.2 & 2 & 0.6 & 3 & 0.9 & 19 & 5.7 \\
\hline Total & 165 & 49.3 & 50 & 14.9 & 120 & 35.8 & 335 & 100 \\
\hline
\end{tabular}

Fuente: elaboración propia. 
temprana edad. Esto corrobora lo dicho por la OIT/ UIP (2002) al afirmar que la pobreza y las prácticas tradicionales de las familias de estos niños y niñas, los obligan a seguir los pasos de sus padres, aprendiendo y practicando un oficio determinado antes de los 12 años de edad, o la creencia en algunas poblaciones de que las niñas no necesitan tanto estudio como los niños.

Según la OIT, en 1997, 250 millones de niños de 5 a 14 años trabajaban, y al menos 120 millones lo hacían a tiempo completo. África registraba la tasa más alta, pues de cada cinco niños de esa edad trabajaban poco más de dos (41 por ciento). En el resto del mundo las proporciones eran las siguientes: América Latina, uno de cada seis (17 por ciento); Asia, uno de cada cinco (21 por ciento), y Oceanía, uno de cada 10 (10 por ciento). (OIT/UIP, 2002, p. 24)

Con el tiempo, los convenios legislados por la OIT y los gobiernos mundiales han variado la edad mínimina de admisión al empleo. En la primera reunión de la Conferencia Internacional del Trabajo, en 1919, se adoptó el primer tratado internacional sobre trabajo infantil, es decir, el Convenio 138 Definición de la Edad Mínima para el empleo (industria), 1919 (núm. 5), que prohíbe el trabajo de niños NNJ de 14 años en establecimientos industriales (OIT/UIP, 2002). Más recientemente, en 1973, se pudo adoptar un convenio completo en la materia que se aplica a todos los sectores económicos y a todos los niños que trabajan, ya sea como asalariados o por cuenta propia, y contiene la definición internacional más completa y autorizada de la edad mínima de admisión al empleo. También es innovador, pues facilita un enfoque flexible y progresivo del problema, sobre todo para los países en desarrollo. El convenio exige a los Estados que lo ratifican la fijación de una edad mínima y define una gama de edades mínimas. Estos mínimos varían según el nivel de desarrollo y según el tipo de empleo y trabajo: no deben ser menores de los 14 años en el período inicial para trabajos ligeros y no menores de 18 años para trabajos riesgosos (OIT/UIP, 2002). Para el decenio de 1990 los convenios fue- ron más agresivos en materia de la edad de ingreso al trabajo, catalogando a todo trabajo en edad escolar como la peor forma de trabajo infantil, ya que interfiere con la educación y la salud física de los niños y las niñas.

En la misma línea, un aspecto importante para resaltar del presente estudio fueron las razones evocadas por los adultos, encargados de los niños y niñas participantes de la muestra, para justificar el ingreso de estos últimos al área laboral. Dichas razones fueron principalmente económicas, afirmando que el trabajo representa "una ayuda para los gastos de la casa". Otra de las razones fue el "gusto del niño y la niña a tener su propio dinero" y, por último, la necesidad de los niños y niñas para financiar su estudio y como estrategia para alejarlos de los vicios. En el estudio realizado recientemente por Amar et al. (2008) en el área de salud mental positiva, la justificación que los padres dieron del ingreso de sus hijos al trabajo desde temprana edad, fue el aporte del trabajo en la construcción de buenos hábitos, la adquisición de responsabilidades y el sentimiento de honra vinculado al ejercicio del trabajo.

En lo referente a la estructura familiar de los niños y niñas víctimas de explotación laboral infantil, se encontró que esta es muy similar a la de los niños y niñas en riesgo. En una proporción significativa, los niños y niñas viven con sus padres biológicos, independientemente de su condición de riesgo o víctima de TI.

Los padres y madres de los niños y niñas víctimas y en riesgo de TI se caracterizan por pertenecer a estratos socioeconómicos bajos, con nivel educativo de básica primaria (en la mayoría de los casos no llegan a $5^{-}$grado) y con experiencia de haber trabajado antes de los 15 años edad.

La falta de preparación profesional, las creencias y valores alrededor del concepto de educación presentes en los padres de los niños y niñas víctimas de TI, ocasiona que estos adultos accedan con facilidad a empleos poco remunerados y eventuales, haciendo necesario que los demás miembros de la familia colaboren con los gastos de la casa y asuman responsabilidades tales como el cuidado de los hijos (más pequeños, menores de un año) y oficios del 
hogar, etc. Mientras los adultos salen a trabajar, los niños y las niñas son quienes asumen estas tareas y en algunos casos acompañan a sus padres en las tareas laborales, fuera del hogar, en condiciones físicas y sociales riesgosas para ellos.

La aparición del TI en la población estudiada aparece asociada a la ausencia de una de las figuras paternas, lo que puede acelerar el proceso de vinculación de los niños y las niñas al trabajo, a fin de poder ayudar con los gastos del hogar. Esto se da en mayor proporción en hogares donde la madre es jefa del hogar, y en menor proporción le siguen aquellos niños y niñas que viven con otros parientes, como abuelos o tíos.

\section{Conclusiones}

En las ciudades de Barranquilla y Santa Marta se presentó mayor porcentaje de niños y niñas en riesgo de explotación laboral infantil. Si bien estos niños no se encuentran laborando, sus condiciones socioeconómicas y familiares son muy similares a las de los niños y niñas víctimas de TI. La cultura, las creencias y valores en torno a la educación y al trabajo a temprana edad siguen siendo los factores detonantes, para que en cualquier momento estos niños y niñas incursionen en el trabajo. Solo es cuestión de tiempo.

Se pudo observar en esta investigación que es mucho lo que a nivel de política social dirigida a la infancia en Colombia se ha realizado, pero falta aplicar una política sostenible y consistente de manera articulada institucionalmente, no solo dirigida a la protección de los niños y las niñas, sino también a sus padres.

La familia es el contexto social fundamental para dar un equipamiento de comportamientos y creencias. Los padres transmiten un amplio espectro de valores y actitudes a sus hijos mediante estilos diversos de comunicación en virtud de los cuales el niño aprende a pensar y a reaccionar de una manera determinada en relación a los acontecimientos y problemas, llegando a generar un modelo comportamental interpersonal. (Amar, 1994, p. 68)
Se puede concluir que, en cualquier situación favorable o desfavorable que ocurra en la niñez, la familia es un factor preponderante para que estas aparezcan o para evitarlas. Es por ello que se necesitan mayores esfuerzos por parte de los centros de investigación de las universidades, las ONG e instituciones gubernamentales, en la elaboración de programas y políticas sociales sostenibles dirigidas a las familias, donde se promueva la restitución y defensa de los Derechos de la Infancia y se hagan propuestas alternativas de desarrollo laboral para los adultos, mitigando el ingreso de niños y niñas al trabajo y garantizando su permanencia en la escuela, dentro de ambientes sociales más sanos.

\section{Referencias}

Amar, J. (1994). Educación infantil y desarrollo social. Barranquilla: Ediciones Uninorte.

Amar, J., Palacio, J., Llinás, H., Puerta, L., Sierra, E., Pérez, A., et al. (2008). Calidad de vida y salud mental en menores trabajadores de Toluviejo. Suma Psicológica, 15(2), 385-404.

Asociación Cristiana de Jóvenes de Bogotá. (2005). Fortalecimiento a familias con niños, niñas y jóvenes trabajadores. Un camino para la prevención y erradicación del trabajo infantil en Colombia (1 1 . ed.) [Versión electrónica: ISBN: 92-2-317460-0]. Bogotá: OIT/Oficina Regional para las Américas/Programa IPEC.

Basu, K. (1998). The economics of child labor. American Economic Review, 88(3), 412-427.

Comité Interinstitucional para la Erradicación del Trabajo Infantil y la Protección del Joven Trabajador. (2008). III Plan nacional para la erradicación del trabajo infantil y la protección del trabajo juvenil 2003-2006. Bogotá: OIT-Colombia.

Consultoría para los Derechos Humanos y el Desplazamiento. (2009, noviembre). Al tablero... Boletín sobre niñez y desplazamiento (No. 2). Descargado el 13 de diciembre, 2009, de http://www.dos-mundos.org/pdf/Boletin\%20ni\%C3\%B1ez\%202.pdf

Departamento Administrativo Nacional de Estadística \& Organización Internacional del Trabajo - Programa Internacional de Erradicación del Trabajo Infantil. (2001). Encuesta Nacional de Trabajo In- 
fantil. Análisis de los resultados de la encuesta sobre caracterización de la población entre 5 y 17 años en Colombia. Disponible en http://www.dane.gov.co/ files/banco_datos/TrabInfantil/OIT_Result_caract_poblacion_5y17.pdf

Departamento Administrativo Nacional de Estadística. (2007). Censo General 2005. Sistema de consulta. Descargado el 5 de julio de 2009, de http://www.dane.gov.co/daneweb_V09/index. php?option $=$ com_content $\&$ view $=$ article $\&$ id $=3$ $15 \&$ Itemid $=124$

Departamento Administrativo Nacional de Estadística. (2008). Análisis en profundidad y términos comparativos de los años 2001, 2003, 2005, 2007 sobre el trabajo infantil. Bogotá: DANE/ICBF. Descargado el 5 de julio, 2009, de http://www. dane.gov.co/files/investigaciones/boletines/ech/ jobinfantil/libro/presentacion.pdf

Díaz, M. (2004). Materiales de trabajo para prevenir y erradicar el trabajo infantil doméstico. Funcionarios que previenen el trabajo infantil doméstico. Guía metodológica [Versión electrónica: 92-2-3156750]. Bogotá: OIT/IPEC Sudamérica.

Flórez, C. \& Méndez, R. (1998). Niños, niñas y jóvenes trabajadores, Colombia 1996. Bogotá: Tercer Mundo.

Guáqueta, C., Virgüez, R., Serrato, L. \& Torrado, M. C. (2001). Niñez y conflicto armado: una mirada institucional al caso colombiano. Bogotá: UN Observatorio sobre Infancia.

Herce, C. \& Torres, B. (1996). Malos tratos a la infancia: reflexiones en torno a la prevención de un problema psicosocial. En C. San Juan Guillén (Coord.), Intervención Psicosocial. Elementos de programación y evaluación socialmente eficaces (pp. 169-205). Bogotá: Anthropos.

Ison-Zintilini, M. \& Morelato-Giménez, G. (2008). Habilidades socio-cognitivas en niños con conductas disruptivas y víctimas de maltrato. Universitas Psychologica, 7(2), 357-367.

Morsolin, C. (2007, 31 de julio). Diferentes miradas sobre el trabajo infantil. ALAI, América Latina en Movimiento. Descargado el 20 de noviembre 2009, de http://alainet.org/active/18852\&lang=es

Muñoz, C. \& Palacios, M. (1978). Aportes al estudio del trabajo infantil en Colombia: Encuesta sobre los niños trabajadores de clase baja en Bogotá. En DANE (2003). Encuesta Nacional de Trabajo Infantil, Noviembre 2001. Bogotá.

Organización Internacional del Trabajo. (2008). Día mundial contra el trabajo infantil 2008: la educación, la respuesta acertada al trabajo infantil. Descargado el 27 de septiembre, 2008, de http:// www.ilo.org/ipec/Campaignandadvocacy/WDACL/2008/lang--es/index.htm

Organización Internacional del Trabajo - Programa Internacional de Erradicación del Trabajo Infantil. (2005). Estadísticas del Trabajo Infantil. Manual sobre las metodologías para la recolección de datos a través de encuestas. Ginebra, Suiza: Programa de Información Estadística y Seguimiento en Materia de Trabajo Infantil (SIMPOC).

Organización Internacional del Trabajo \& Unión Interparlamentaria. (2002). Guía práctica para Parlamentarios (No. 3). Erradicar las peores formas de trabajo infantil. Guía para implementar el Convenio número 182 de la OIT. Ginebra, Suiza: Autores.

Ordóñez, P. \& Álvarez, L. (2006). Derechos de la niñez e intervención frente al trabajo infantil en Colombia: análisis de caso. Bogotá: UN Observatorio sobre Infancia. Descargado el 3 de noviembre, 2009, de http://www.observatorioinfancia.unal.edu.co/ investigacion.html

PARTNERS of the AMERICAS, DevTech Systems, Fundación Centro Internacional de Educación y Desarrollo Humano \& Mercy Corps. (2007). Proyecto Eliminación del trabajo infantil, a través de la educación en Colombia, "Edúcame primero, Colombia" (Acuerdo de Cooperación No. IL16574-07-75K). Bogotá: Autores.

Pinzón, A., Briceño, L., Gómez, A. \& Latorre, C. (2003). Trabajo infantil en las calles de Bogotá. Revista Ciencias de la Salud, 1(2), 151-63.

Programa Interdisciplinario de Apoyo a la Comunidad. (2003). Del socavón a la vida. Linea base para el acercamiento a la erradicación y prevención del trabajo infantil en la minería artesanal colombiana. Bogotá: MINERCOL/PNUD. Disponible en http:// www.bdigital.unal.edu.co/1283/2/01PREL01.pdf

Rey, C. (2004). Respuestas sociales ante situaciones hipotéticas de tensión interpersonal de un grupo de niños y niñas institucionalizados por maltrato 
Vanessa Romero, José Amar, Jorge Palacio, Camilo Madariaga, Eloísa Sierra, Sandra Quintero

físico y de un grupo de niños y niñas no maltratados. Universitas Psychologica, 3(2), 165-178.

Sandoval, A. (2007). Trabajo infantil e inasistencia escolar. Revista Brasileira de Educação, 12(34), 68-80. 